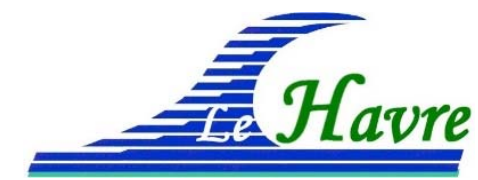

\title{
Une passe microtidale intermittente contrôlée par le vent : l'exemple du grau de La Franqui (Occitanie)
}

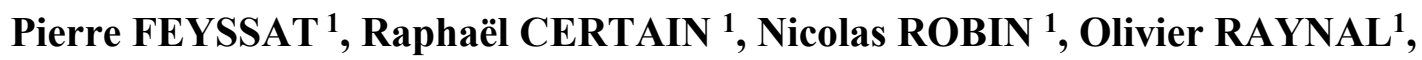 Nicolas ALEMAN ${ }^{1}$, Bertil HEBERT ${ }^{1}$, Koffi Franc-Xavier ADOU ${ }^{1}$, Samuel MEULE ${ }^{2}$, Frédéric BOUCHETTE ${ }^{3}$, Damien SOUS ${ }^{4}$}

1. CEFREM, Université de Perpignan Via Domitia, 52 Avenue Paul Alduy, 66000 Perpignan, France.pierre.feyssat@univ-perp.fr

2. Marseille Université, CNRS, IRD, Collège de France, CEREGE, Aix-en-Provence, France.

3. Géosciences-Montpellier, Université de Montpellier, Place Eugène Bataillon, 34095 Montpellier, France.

4. MIO-Seatech, Université de Toulon, 83041 Toulon, France.

\section{Résumé :}

Ce travail porte sur la caractérisation du fonctionnement d'une des dernières passes intermittentes non-endiguées de Méditerranée française : le grau de La Franqui (Aude). L'étude consiste en un suivi morphologique régulier de la plage pendant 2 ans avec un DGPS-RTK. Le déploiement d'instruments hydrodynamiques (capteurs de pression et courantomètre) sur 2 mois permet aussi de mesurer les variations de niveau d'eau à la côte et dans la lagune en fonction des conditions de forçages hydro-météorologiques.

Mots-clés : ICOLL, Passe microtidale, Influence du vent, Lagune côtière, Golfe du Lion

\section{Abstract:}

This work focuses on characterising the functioning of one of the last non-diked intermittent inlets in the French Mediterranean: the grau de La Franqui (Aude). The study consists of regular morphological surveys of the beach for more than 2 years with a DGPS-RTK. The deployment of hydrodynamic instruments (pressure sensors and current meter) over 2 months allows us to measure the water level variations on the coast and in the lagoon according to the hydro-meteorological forcing conditions.

Keywords: ICOLL, Microtidal inlet, Wind dominated, Coastal lagoon, Gulf of Lion

\section{Introduction}

Les passes microtidales intermittentes sont référencées dans la littérature sous le terme d'ICOLL (Intermittently Closed/Open Lakes and Lagoons). Ce sont généralement des passes dynamiques caractérisées par des phases d'ouverture/fermeture périodiques de leurs exutoires entre la mer et une lagune côtière (HAINES et al., 2006). Elles sont courantes dans les environnements microtidaux dominés par l'énergie des vagues où d'importantes variations dans les volumes des décharges fluviales et dans les régimes de 


\section{Thème 2 - Dynamique sédimentaire}

houles sont observées. Le modèle de fonctionnement le plus répandu décrit des ouvertures causées par des augmentations du niveau d'eau dans la lagune suite à de fortes décharges fluviales ayant pour effet de rompre le cordon littoral. Les fermetures sont causées par un apport de sable marin lorsque le flux sortant diminue d'intensité (COOPER, 1994 ; HAYES \& FITZGERALD, 2013 ; MCSWEENEY et al., 2017). Cette étude s'intéresse à la dynamique de la passe microtidale intermittente de la lagune de La Palme. Les décharges fluviales étant trop faibles pour expliquer à elles seules les ruptures du cordon littoral, le but est alors de comprendre les mécanismes contrôlant le fonctionnement de ce système.

\section{Site d'étude}

Le grau de La Franqui est situé dans le Golfe du Lion, le long du littoral du Narbonnais au nord du cap Leucate (Aude). Cette passe permet la communication entre la lagune de La Palme (surface 500 ha, volume $2.6 \mathrm{M} \mathrm{m}^{3}$ d'eau) et la mer Méditerranée. C'est un milieu microtidal (marnage moyen de $0,3 \mathrm{~m}$ ) où le vent de terre (Tramontane) est dominant (60\% du temps, vitesse pouvant atteindre $\left.30 \mathrm{~m} \cdot \mathrm{s}^{-1}\right)$. Le vent marin est moins fréquent (30\% du temps) mais s'accompagne d'une agitation du plan d'eau pouvant générer de violents épisodes de tempêtes hivernaux (figure 1).

La dérive littorale est localement dirigée vers le nord au niveau du site d'étude. À l'échelle du Golfe du Lion, elle est orientée vers le nord dans la partie sud et vers le sud dans la partie nord. Les deux dérives se rencontrent au niveau du littoral du Narbonnais (où se trouve notre zone d'étude) et assurent un important disponible sédimentaire. L'avant-côte présente un système à deux barres festonnées (ALEMAN et al., 2015) de type RBB (Rythmic Bar and Beach).

\section{Matériel et méthodes}

\subsection{Suivi morphologique}

Un suivi morphologique de la plage à une fréquence mensuelle est effectué depuis novembre 2017 à l'aide d'un DGPS-RTK centimétrique (figure 1) suivant des transects transversaux (espacés de $10 \mathrm{~m}$ ) et longitudinaux (trait de côte et haut de berme). Cette démarche permet la réalisation de Modèles Numériques de Terrain (MNT) sur ArcGis avec la méthode d'interpolation Voisins Naturels (précision en $Z$ de l'ordre de $\pm 2,5 \mathrm{~cm}$ ). En tout, 20 MNT ont été générés et utilisés pour cette étude.

\subsection{Suivi hydrodynamique et météorologique}

Deux capteurs de pression (RBR Virtuoso) et un courantomètre (NORTEK Vector) ont été déployés simultanément sur le site d'étude (lagune et mer) entre le 7/02/2019 et le 27/03/2019 afin d'estimer les variations des hauteurs d'eau à différents endroits clé du système (figure 1). 


\section{XVİ̀mes Journées Nationales Génie Côtier - Génie Civil \\ Le Havre 2020}

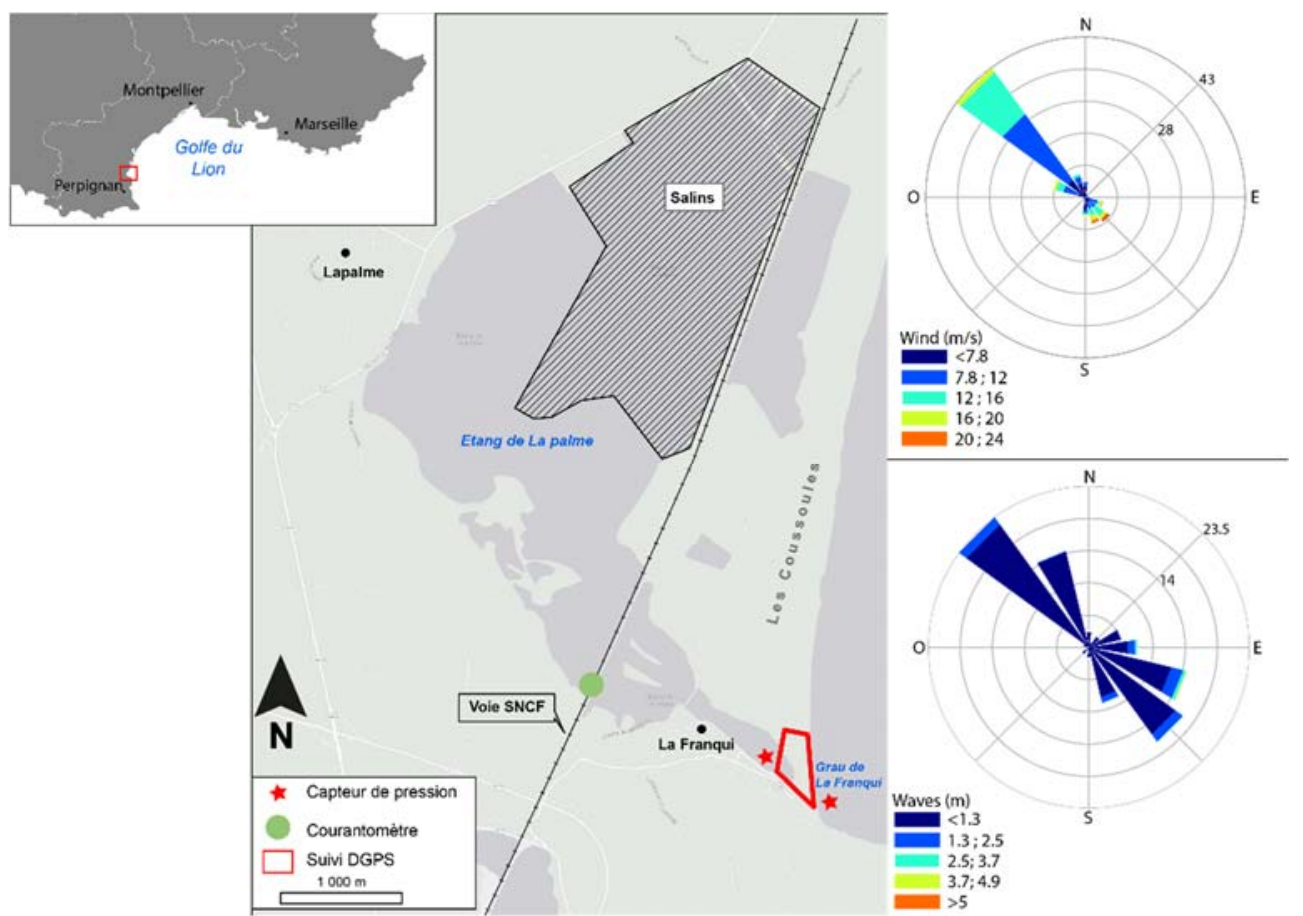

Figure 1. Localisation du site d'étude et plan de positionnement des instruments.

Concernant l'enregistrement de la houle, il est effectué par la bouée CANDHIS de Leucate (CEREMA / DREAL LR) située au large de la zone d'étude (40 m de profondeur). Enfin, les données de vents proviennent de la station Météo France du sémaphore de Cap Leucate située à environ 2,5 km à l'est de la plage de La Franqui, à l'extrémité du cap à une altitude de $42 \mathrm{~m}$.

\section{Résultats et interprétation}

Le suivi morphologique effectué depuis 2 ans a permis de distinguer différentes configurations du grau en fonction des forçages dominants. La Tramontane apparaît comme un paramètre prédominant dans le fonctionnement du système. Son action entraîne un basculement du plan d'eau lagunaire induisant une accumulation d'eau le long de la barrière littorale et une légère décote en mer lors des phases les plus intenses (voir figure 2 avec les événements du 28/02, 01/03 et 03/03). En parallèle, elle décape une partie du sable superficiel de la plage et l'exporte vers la mer où il va se déposer sur la proche avant-côte. Durant ces événements, l'influence de la houle est non significative avec la présence d'un plan d'eau très peu énergétique. Les cornes de sinuosités de plage vont s'abaisser et s'étaler entrainant une avancée du trait de côte. 


\section{Thème 2 - Dynamique sédimentaire}

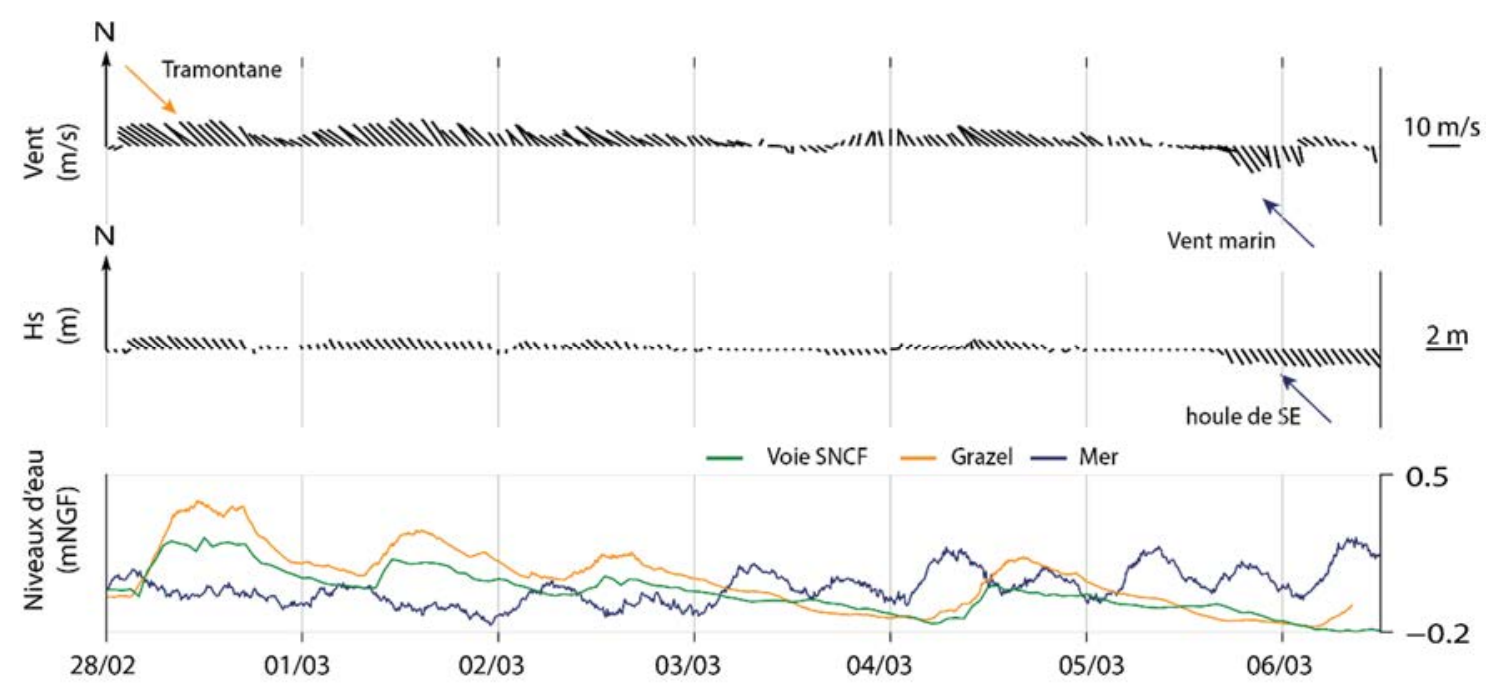

Figure 2. Niveau d'eau en mer et dans la lagune.

L'ouverture du grau se fait suite à plusieurs épisodes intenses de Tramontane associés à un contexte morphologique de la plage particulier en raison du décapage éolien intense caractérisé par une berme moins haute et en pente plus douce vers la mer au niveau des cornes de festons (figure 3a). Ces cornes sont des zones qui connectent la plage et le système de barres d'avant-côte festonnées (figure 4). Ce point de fragilité, en raison de la faible altitude de la berme, associé à l'effet de la surcote dans la lagune et du clapot qui déferle à l'arrière du cordon, favorise l'ouverture de la passe. L'ouverture entraîne la vidange de l'eau lagunaire et l'expulsion du sédiment sous forme d'un delta en mer (figure $3 \mathrm{~b}$ ). Le courant sortant est alors intense, proche de $1 \mathrm{~m} \cdot \mathrm{s}^{-1}$. Dans les cas de surcotes très importantes liées à un vent particulièrement violent, des ouvertures avec plusieurs passes peuvent être observées. Un second mécanisme d'ouverture beaucoup moins fréquent peut avoir lieu pendant les tempêtes marines les plus intenses $(\mathrm{Hs}>3 \mathrm{~m})$ où la submersion importante de l'arrière-plage par de l'eau marine créé les conditions à la rupture du cordon sableux. Concernant les phases de comblements de la passe, elles semblent être contrôlées par l'action des petites houles d'est de hauteur décimétriques à forte période (autour de 10s) liée au vent marin. Ces conditions hydrométéorologiques favorisent le remaniement du sédiment de l'avant-côte (déposé lors de l'ouverture), entrainant la formation d'un banc de sable qui va s'accoler au trait de côte (figure $3 \mathrm{c}$ ). Si le courant sortant est suffisamment faible $\left(<0,1 \mathrm{~m} \cdot \mathrm{s}^{-1}\right)$, cette remontée de sédiment obstrue l'exutoire de la passe. 


\section{XVİ̀mes Journées Nationales Génie Côtier - Génie Civil \\ Le Havre 2020}

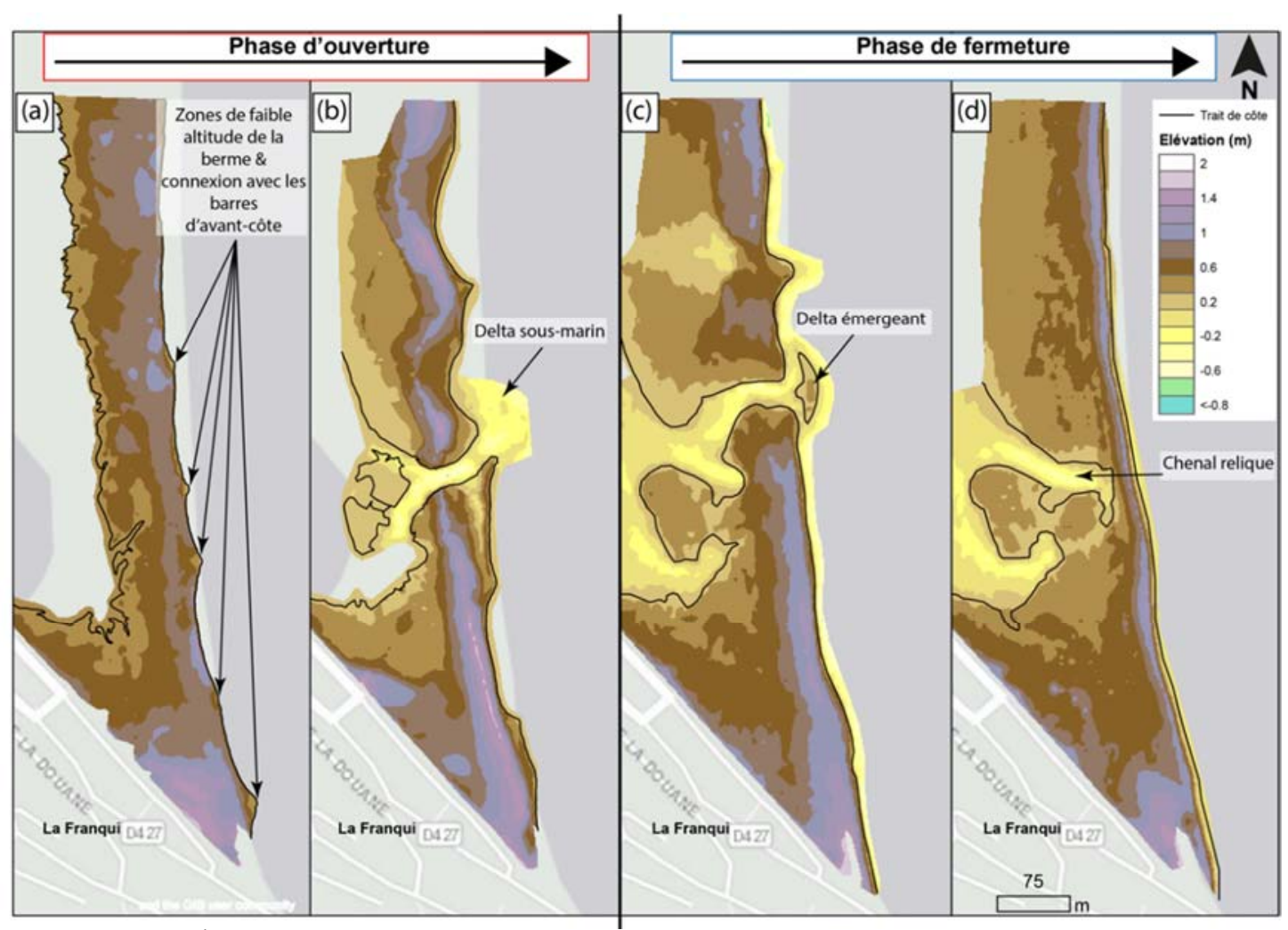

Figure 3. Évolutions morphologiques de la plage au cours d'un cycle ouverture / fermeture.

Les tempêtes sont courantes en hiver et s'accompagnent de fortes houles dirigées vers la côte et de précipitations. Le trait de côte enregistre alors une rétrogradation pouvant atteindre plusieurs dizaines de mètres, particulièrement visible au niveau des cornes des festons. Les sinuosités de la plage ne sont alors presque plus visibles, la pente du talus de collision est subverticale et la berme gagne plusieurs dizaines de centimètres de hauteur. Des cônes de débordement peuvent se mettre en place en lien avec la surcote et des processus de swash importants. Dans le cas où la passe est déjà établie au moment de la tempête, le chenal sera creusé et élargi par les courants y circulant.

Ainsi, le suivi morphologique permet d'illustrer un schéma d'évolution du système sur un cycle saisonnier qui s'avère original au regard de la bibliographie existante. La première ouverture en début d'hiver est liée à l'apparition de fortes conditions de vent de terre dominant (Tramontane). Au cours de l'hiver, des phases rapides de fermetures et de réouvertures peuvent aussi se mettre en place avec l'alternance des forçages hivernaux. La durée des fermetures est trop courte pour que la plage revienne à un état similaire à celui de sortie d'été (figure 3d). La fragilité de la berme facilite alors l'ouverture lors de prochains épisodes de Tramontane. Les conditions printanières moins énergétiques permettent de clôturer le cycle hivernal avec la fermeture de la passe à la fin du printemps, et ce, pendant l'ensemble de la période estivale. 


\section{Thème 2 - Dynamique sédimentaire}

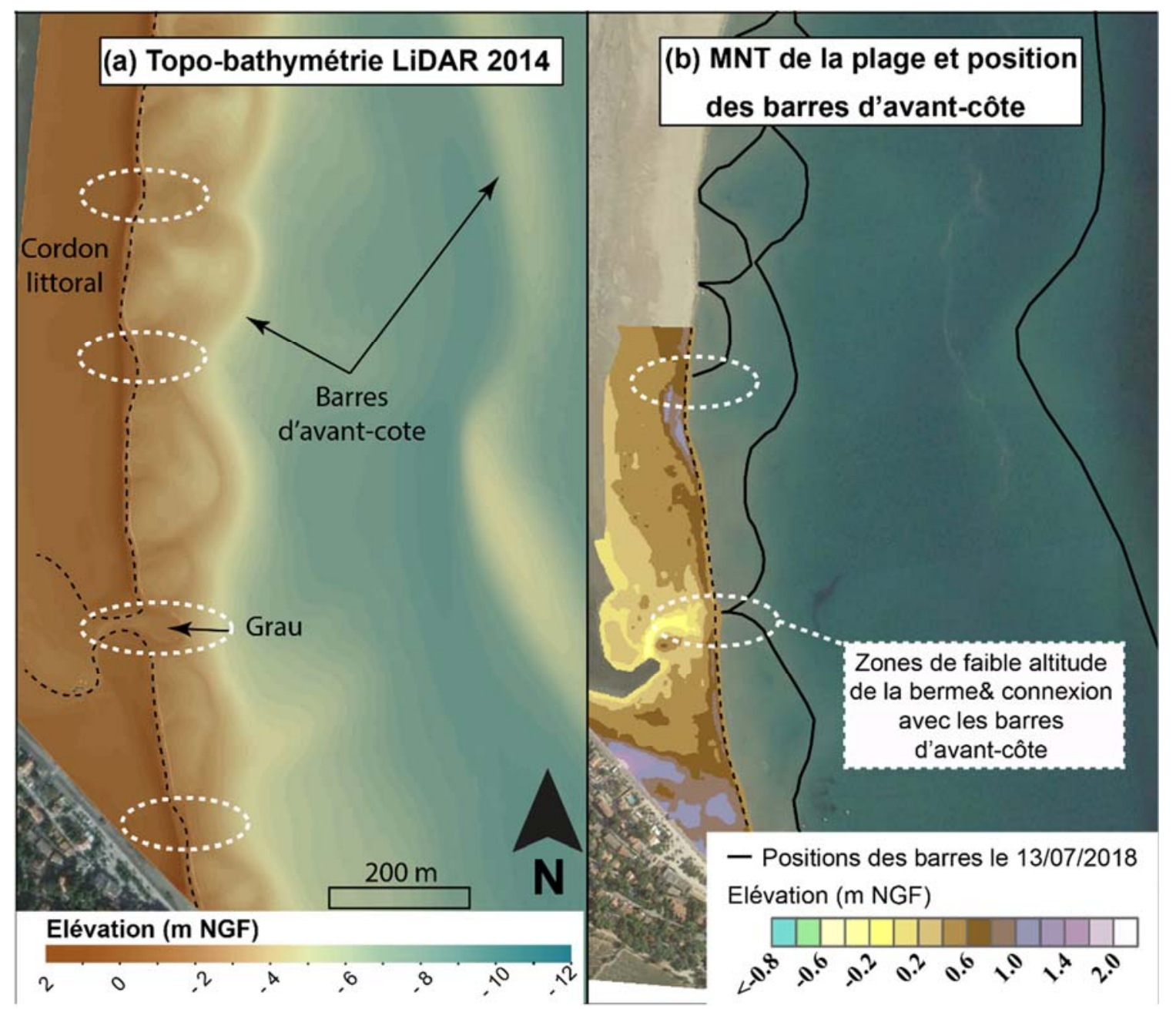

Figure 4. LiDAR montrant les connections entre la berme et le système interne de barres,(a) après ouverture, (b) avant ouverture.

\section{Discussion}

\subsection{Des processus de fonctionnements complexes}

Les ouvertures se font majoritairement en conditions de Tramontane, plus rarement à la suite de tempête et les fermetures se font à la suite de périodes de petites houles dirigées vers la côte.

Cependant, ces processus sont aussi tributaires d'un contrôle morphologique complexe de la plage. En effet, la position du grau est guidée par la position des cornes de plage qui connectent la plage aérienne au système de barres internes. La dynamique même de ces systèmes de barres et leurs interconnections avec la plage aérienne sont mal compris. De plus, on observe que les ouvertures au cours de l'hiver suivent préférentiellement le cours des anciens chenaux qui n'ont pas eu le temps d'être comblés. Cette complexité dans les mécanismes de contrôles entraîne le passage par de nombreuses phases morphologiques transitoires de la barrière. 


\section{XVI èmes Journées Nationales Génie Côtier - Génie Civil \\ Le Havre 2020}

\subsection{Comparaison avec les modèles existants}

Le mécanisme de fermetures du grau se rapproche fortement du modèle de fonctionnement de cross-shore transport des ICOLL (COOPER, 1994). Les ouvertures contrôlées par le vent ne sont cependant pas décrites dans la littérature pour les systèmes d'ICOLL. Ces ouvertures sont décrites comme faisant suite à de fortes décharges fluviatiles (COOPER, 1994 ; HAYES \& FITZGERALD, 2013). Le forçage éolien doit aussi jouer un rôle important sur d'autres passes intermittentes dans les zones microtidales où les vents de terre sont importants, comme sur la façade nord méditerranéenne par exemple. En revanche, cela semble difficilement s'appliquer si le site est fortement aménagé (digues, enrochement ...) dans le but de maintenir une ouverture permanente.

\section{Conclusion}

Le grau de La Franqui pourrait être un nouveau type d'ICOLL, car les passes microtidales intermittentes contrôlées par le vent et la position des barres d'avant-côte ne sont contenues dans aucune des classifications actuelles.

Le fonctionnement du grau est régi par des processus complexes avec des effets de rétroaction importants. L'équilibre des forçages actuels pourrait changer dans le futur avec l'augmentation prévue du niveau marin ou un changement dans le régime des vents. De plus, le système est très résilient du fait des importants apports sédimentaires dus à la convergence des dérives littorales. Mais il est possible que cela change à l'avenir à cause de la diminution des apports fluviaux et des aménagement portuaires prévus au nord en amont dérive.

\section{Remerciements}

Les auteurs tiennent à remercier le PNR de la Narbonnaise en Méditerranée (https://www.parc-naturel-narbonnaise.fr/) pour son soutien financier. La plateforme GLADYS (http://www.gladys-littoral.org/en) pour le prêt de matériel et l'appui technique. Le site de La Franqui fait partie du réseau d'observation SNO DYNALIT (https://www.dynalit.fr/).

\section{Références bibliographiques}

ALEMAN N., ROBIN N., CERTAIN R., ANTHONY E.J., BARUSSEAU J.P. (2015). Longshore variability of beach states and bar types in a microtidal, storm-influenced, low-energy environment. Geomorphology, Vol. 241, pp 175-191. https://doi.org/10.1016/j.geomorph.2015.03.029

COOPER J.A.G. (1994). Lagoons and microtidal coasts. In: Carter, R.W.G., Woodroffe, C.D. (Eds.), Coastal Evolution Late Quaternary Shoreline Morphodynamics, pp 219-265. 
Thème 2 - Dynamique sédimentaire

HAINES P.E., TOMLINSON R.B., THOM B.G. (2006). Morphometric assessment of intermittently open/closed coastal lagoons in New South Wales, Australia. Estuarine, Coastal and Shelf Science, Vol. 67, pp 321-332. https://doi.org/10.1016/j.ecss.2005.12.001

HAYES M.O., FITZGERALD D.M. (2013). Origin, Evolution, and Classification of Tidal Inlets. Journal of Coastal Research, Vol. 69(sp1), pp 14-33. https://doi.org/10.2112/si_69_3 MCSWEENEY S.L., KENNEDY D.M., RUTHERFURD I.D., STOUT J.C. (2017). Intermittently Closed/Open Lakes and Lagoons: Their global distribution and boundary conditions. Geomorphology, Vol. 292, pp 142-152. https://doi.org/10.1016/j.geomorph.2017.04.022 